\title{
VISUOMOTOR TRACKING WITH DELAYED VISUAL FEEDBACK
}

\author{
R. C. Miall, D. J. WeiR* and J. F. Stein \\ University Laboratory of Physiology, Parks Road, Oxford OX1 3PT; *Engineering in Medicine \\ Laboratory, Imperial College, London SW7 2AZ
}

\begin{abstract}
A rhesus monkey and five human subjects used a hand-held jnystick to track unpredictable continuously moving targets. Both monkey and human respond by making discrete ("step-and-hold") corrections of positional error, at an average frequency of 1.33 and 2.26 movements/second, respectively. By delaying visual feedback of joystick position, we could reduce these frequencies in a predictable manner.

These results imply that the primate visuomotor system probably does not operate as a "sampled-data mechanism" governed by an asynchronous clock, but that inevitable delays in visuomotor feedback control determine the frequency of corrective movements.
\end{abstract}

The idea that humans performing tracking tasks can be viewed as asynchronous sampled-data control systems has been in the literature for many years. ${ }^{2,3,13}$ In tasks involving tracking unpredictably moving targets, step movements at unpredictable times or very slow ramp movements of the target, subjects don't track smoothly but make positional error corrections only intermittently. Hence the track followed tends to have a discrete step-and-hold pattern, and there is a minimum average period between corrective movements. It is generally assumed that some form of asynchronous clock or timer is involved in this "sampling" process. The frequency of corrections is about 1-3 per second in humans ${ }^{2,3,9,13}$ and 1-2 per second in trained monkeys. ${ }^{8}$ Predictable waveforms are followed smoothly, however, and in humans tracking sinusoids, very few corrective steps can be seen. ${ }^{10}$

The nature of the sampling mechanism responsible for intermittent movements remains obscure. Navas and Stark' suggested that refractoriness of alpha motorneurones may be involved, but this has been disproved. ${ }^{1}$ We have suggested that intermittent control may result not from the operation of a "sampler" but from intermittent flow of information around the visuomotor feedback loop. ${ }^{7}$ This implies that the rate of corrections should be controlled mainly by delays in the loop (visual processing, motor programming and movement execution); hence, no explicit sampling mechanism would be required. If the rate of corrective movements is controlled in this way, then any additional delays imposed on the feedback loop would decrease the correction rate by a predictable amount. The normal feedback loop time may be estimated by the reciprocal of movement frequency;

Address for correspondence: R. C. Miall, University Laboratory of Physiology, Parks Road, Oxford OX1 3PT, U.K.

Abbreviation: m.p.s., movements per second. the predicted movement frequency is then the reciprocal of normal loop time plus added loop delays.

We report here the results of experiments designed to test this hypothesis in a visual tracking task, by increasing the time delay between corrective movements and visual feedback of these movements, in both a trained rhesus monkey and in five human subjects.

\section{EXPERIMUNTAL PROCEDURTS}

The same experimental equipment was used for both the human and monkey trials. The subject sat in front of an oscilloscope screen on which a circular target was displayed (diameter: $1 \mathrm{~cm}$ for monkey, $0.5 \mathrm{~cm}$ for humans). The target could be moved horizontally over a $6 \mathrm{~cm}$ range by a function generator. Slow sinusoids are effectively unpredictable to monkeys ${ }^{8}$ and a series of six frequencies between 0.036 and $0.46 \mathrm{~Hz}$ was used. The monkey was also tested tracking a pseudo-random waveform: an RML $380 Z$ lab computer was programmed to sum 16 non-harmonic sines of equal amplitude but random phase. The resultant waveform had a frequency range of $0.039-0.69 \mathrm{~Hz}$, and it repeated in approx. $1 \mathrm{~min}$. Humans can predictively follow sinusoids as low as $0.1 \mathrm{~Hz}$, however, ${ }^{10}$ so the human subjects were tested with the pseudo-random waveform only.

The subject was required to use a free-moving joystick to control the X-Y position of a small monitor spot also displayed on the oscilloscope screen; the spot thus provided visual feedback of position. A 2-channel analogue delay (based on the Reticon SAD1024 i.c.) could be switched-in between the joystick and display, so that visual feedback could be delayed by up to $500 \mathrm{~ms}$.

The monkey was trained over several months to maintain the monitor spot within the target circle. He was rewarded with apple juice if he remained on-target for about half a second. Five human subjects were given several minutes practice at the task before their responses were recorded. They were not told about the delay, and delay times were randomly selected at $2 \mathrm{~min}$ intervals, separated by $1 \mathrm{~min}$ at zero delay. Delays of less than $100 \mathrm{~ms}$ proved to be difficult for the subjects to detect, but long delays were more obvious. Subjects reported afterwards that delays between 100 and $200 \mathrm{~ms}$ gave the joystick a rather "loose" feel. Longer delays were very difficult to overcome, and two of our subjects complained that with delays of $300 \mathrm{~ms}$, the equipment was "not working properly". 
The target and joystick position signals were recorded on magnetic tape. These were then displayed on a chart recorder, along with the electronically-derived velocity signal. The subjects made clear step-and-hold corrective movements while tracking the target waveforms, and these were particularly noticeable as discrete peaks in the velocity record (Fig. 1b). The average number of movements per second (m.p.s.) was determined for each subject with immediate visual feedback and when feedback delays of $50-300 \mathrm{~ms}$ (humans) or $50-500 \mathrm{~ms}$ (monkey) were introduced. First, the number of movements made in 1 min periods were counted. A "movement" was taken to be a single, smooth change of position, and hence a single peak in the velocity record. Twenty records from human subjects were counted by two observers to gauge the reliability of the estimates. The technique was also tested for several of the monkey's records by spectral analysis of the sinusoidal tracking records. In any onc record the rate of corrections was quite variable: the subject tended to make more frequent movements when the target was moving fastest but there was also variation about the mean rate which was not related to the target trajectory. Fourier analysis was then unable to detect a clear component at the average frequency of corrective movements (Fig. 1c). A frequency spectrum was therefore plotted by averaging $20-30$ successive autoregressive spectral estimates of the movement record, each calculated from 256 points $(3.6 \mathrm{~s}$, or $125 \%$ of the target period) triggered with reference to a fixed position in the target cycle? This technique allowed us to isolate the average frequency component which was due to the stepand-hold movements (Fig. 1d).

The predicted fall in movement rate $\left(R_{d}\right)$ when delays are introduced is hyperbolic, and can be determined by:

$$
R_{\mathrm{d}}=1.0 /\left(1.0 / \mathbf{R}_{0}+\mathrm{d}\right)
$$

where $R_{0}$ is the movement rate with no delay, and $d$ is the introduced delay (see Introduction). This equation is used to plot the expected fall in movement rate shown in Figs 3, 4, 6 and 9.

However, the results of spectral analysis indicated that the fall was twice that predicted by equation 1 . This is because the spectral technique measures movement cycles, and each cycle is made up of two movements. Hence, the frequency of movements $\left(f_{d}\right)$ is predicted by:

$$
f_{d}=1.0 /\left(1.0 / f_{0}+2 d\right)
$$

where $f_{0}$ is the movement frequency with no delay.

Step-and-hold tracking is a form of amplitude modulation of the target waveform. The velocity signal can be idealized as a pulse-amplitude modulated signal (e.g. Ref. 5). The spectrum of such a P.A.M. signal would not contain a frequency component at $\mathrm{f}_{0}$, but would have "side-band" components $\left(f_{1}\right.$ and $\left.f_{2}\right)$ at $f_{0}+f_{v}$, where $f_{t}$ is the target frequency. Therefore we modified equation 2 to:

$$
\mathrm{f}_{\mathrm{d}}=1.0 /\left(1.0 / \mathrm{f}_{0}+2 \mathrm{~d}\right) \pm \mathrm{f}_{\mathrm{t}}
$$

and $f_{0}$ must be estimated by:

$$
\left(f_{1}+f_{1}\right) \text { or }\left(f_{2}-f_{1}\right)
$$

This equation is therefore used to plot the expected frequency components in Fig. 5.

\section{RESULTS}

\section{Monkey experiments}

A typical example of the monkey's tracking responses is shown in Fig. Ia. With no delay of visual feedback clear step-and-hold movements can be seen, and the average rate of these corrective movements ranged from 1.11 to 1.62 movements per second (m.p.s.: mean 1.33). There was a slight decrease in the rate of positional corrections as target frequency increased (Fig. 3), although the scatter was considerable. Introduction of delays in the visual feedback of position caused two changes in the monkey's responses. First, he made less frequent corrections of position, so that the average frequency of movements fell as feedback delay increased (Fig. 2b). Second, he increasingly tended to overshoot the target; with delays of $200 \mathrm{~ms}$ or more this resulted in clear "hunting" about the target sine-wave (Figs 2b and c).

The hyperbolic fall in rate of corrective movements as feedback delay increased is shown in Fig. 3, for three target frequencies. The data at cach target frequency were fitted by a curve calculated by addition of the introduced delay to the average period of movements when working without delay (see Experimental Procedures). In order to plot all 6 frequency curves together, the data sets were normalized to the average base rate with no feedback delay of 1.33 m.p.s. Predicted values then all fall on a single curve, and the experimental results for all target frequencies are shown together with this predicted curve in Fig. 4. It can be seen that there is very close agreement between observed and predicted results.

Fourier analysis was not of use in confirming these findings (Fig. 1c), because the rate of corrections varied about an average value, both within the target cycle and over several cycles. Using a spectral estimation technique we were able to determine spectral estimates for much shorter data lengths, and by triggering these data windows from a set point in the target cycle, we calculated an average spectrum that showed a number of components other than at the target frequency (Figs 1d and 5). These appeared in many of the spectra as a complex of three peaks, separated by the difference between the target frequency and the average movement frequency, and moved together towards lower frequencies as feedback delays were introduced. Evidence for such reflected peaks was found in many of the individual spectra plotted, and can also be seen in the averaged spectra (Fig. 5). The second peak $\left(f_{2}\right)$ was often of small amplitude and is not evident in all the averaged spectra. The target frequency in Fig. 5 is $0.35 \mathrm{~Hz}$, so $f_{0}$ is situated at $\left(f_{1}+0.35\right)$, i.e. at $1.4 \mathrm{~Hz}$. The predicted locations of $f_{d}, f_{1}$ and $f_{2}$ have been determined by substituting this value in equation 3 (Experimental Procedures). Many of the peaks in Fig. 5 are close to their predicted locations.

With the longest delays, 200 and $300 \mathrm{~ms}$, the monkey showed periods of overt "hunting" about the target sinusoid. These records are therefore described in part by the amplitude modulation considered above, but in other parts are better described by frequency addition. The averaged spectra for these long delay trials therefore contain the two "sideband" peaks predicted by equation 3 , but also a peak at $f_{\mathfrak{d}}$. 


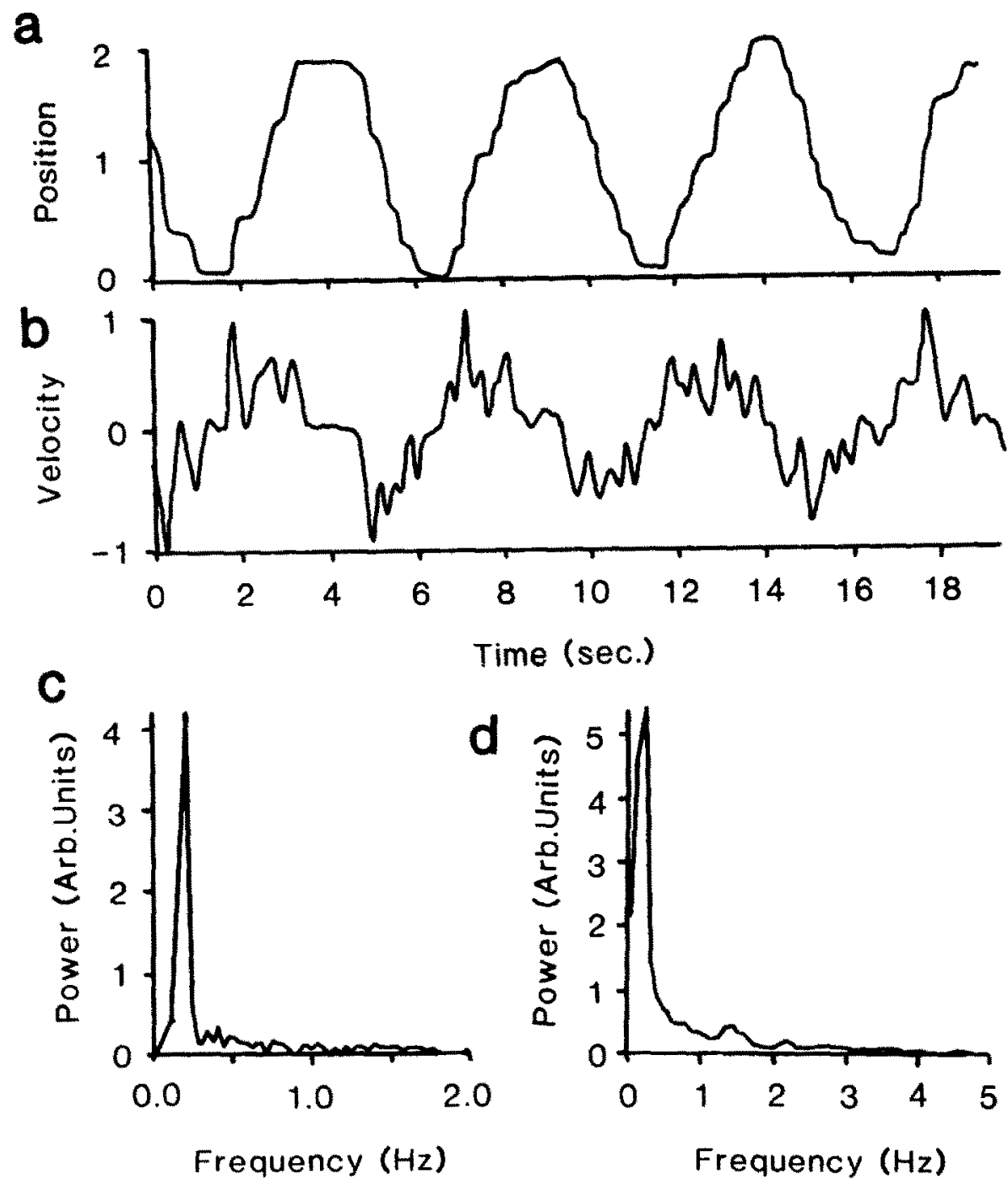

Fig. 1. Response of a monkey tracking a $0.2 \mathrm{~Hz}$ sinusoidal target (not shown). Step-and-hold movements can be seen in the position record (a), and appear as discrete peaks in the velocity record (b). c: 1024 point FFT power spectrum of Fig. la. The large peak is at the target frequency, little power is associated with the step-and-hold movements. d: an average autoregressive spectrum calculated by averaging twenty 256 point spectra triggered off the target signal; note the peaks at 1.5 and $2.2 \mathrm{~Hz}$.

The monkey's responses to the pseudo-random waveform were very similar to his responses to sinusoidal targets. The rate of corrective movements when tracking the pseudo-random waveform were also similar, and fell as predicted when feedback delays were introduced (Fig. 6).

\section{Human experiments}

The humans' responses to the pseudo-random target were similar to those of the monkey, with clear signs of step-and-hold positional corrections in all 5 records (Fig. 7). As with the monkey, there was considerable variation in the moment-to-moment frequency of movements, but averaged over 1 full cycle of the target function (c. $60 \mathrm{~s}$ ) they ranged from 2.01 to 2.47 m.p.s. (mean 2.26). In order to assess the errors introduced by visual identification of "movements", 20 records from 2 of the subjects were counted by 2 observers. The difference in numbers of movements counted by the 2 observers was only $4.1 \%$ $( \pm 1.91 \% \mathrm{SE})$, while the correlation between their counts was 0.93 .

As before, introduction of delays reduced the number of movements made (Fig. 7) and increased their amplitude. Brief periods of hunting about the target were seen at high feedback delays (Fig. 8a), but these were not as pronounced as in the monkey's responses. However, unlike the monkey, the human subjects' movement frequency did not continue to fall with delays higher than about $150 \mathrm{~ms}$, and for longer delays the frequency increased again (Fig. 9). This was true of all 5 subjects. The main cause for this 

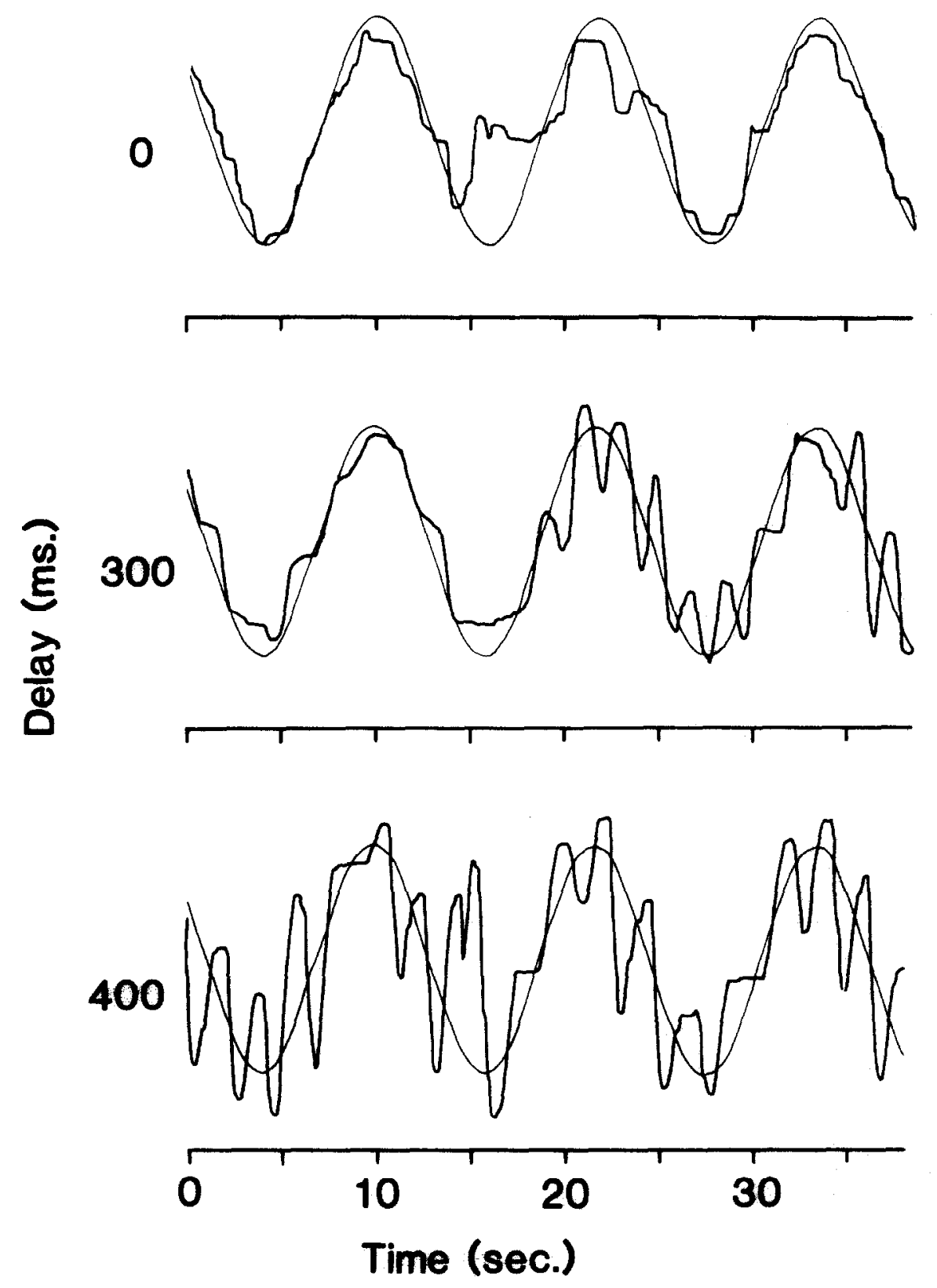

Fig. 2. Typical tracking responses of a trained monkey (heavy lines), tracking a horizontal sinusoid (light lines) with immediate visual feedback (upper trace). Introduotion of visual feedback delays of 300 and $400 \mathrm{~ms}$ reduced the frequency of the corrective movements (left, middle trace) and caused "hunting" about the target sinusoid (right, middle and lower traces).

increase was brief periods of very fast corrections. In some of these periods the tracking bocame quite smooth, and it became difficult to distinguish individual peaks in the velocity waveform (Fig. 8b).

No frequency analysis of the human responses was attempted because of the difficulties in interpreting the complex spectra generated by the pseudo-random target function.

\section{Discussion}

Both the monkey and human subjects tracked the target by making discrete step-and-hold movements.
The frequency of positional corrections was variable, averaging 1.33 and 2.26 movements per sooend (m.p.s.) for monkey and humans, respoctively. The slight decrease in the monkey's rate of corrections as the target frequency increased is not folt to be significant. Other experiments have shown that movement rate increases slightly as frequency increases, but only doubles in rate as target hoquency increases 30-fold. ${ }^{8}$

Minimum reaction times are in the range 200-250 ms for both monluoys" and humins, "it However, in a tracking task calculating positional error 


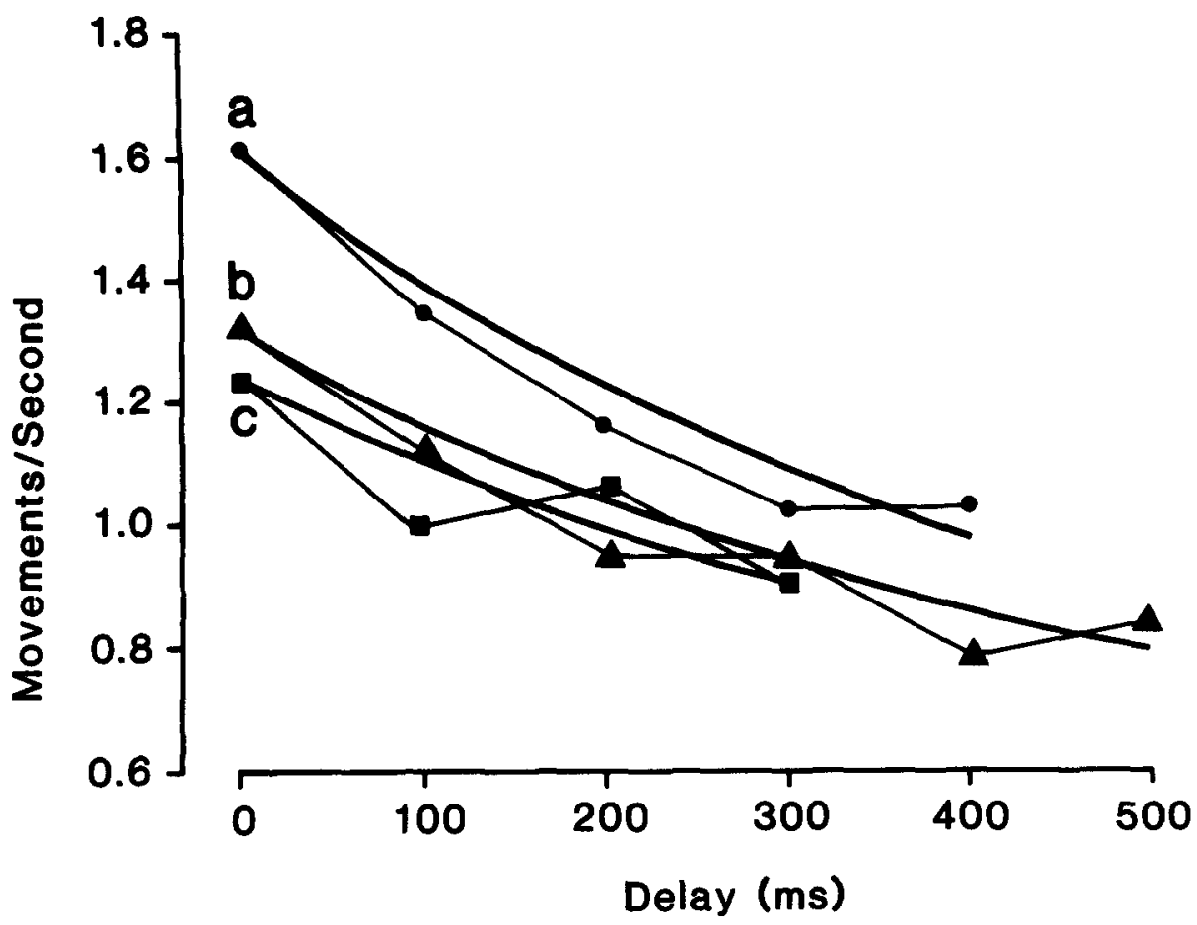

Fig. 3. Average rate of monkey's corrective movements when tracking sinusoids, with additional delays in visual feedback. Target frequency: a: $0.086 \mathrm{~Hz} ; \mathrm{b}: 0.036 \mathrm{~Hz} ; \mathrm{c}: 0.2 \mathrm{~Hz}$. The predicted fall in movement rate for each frequency (heavy line) was determined using equation 1 (Experimental Procedures).

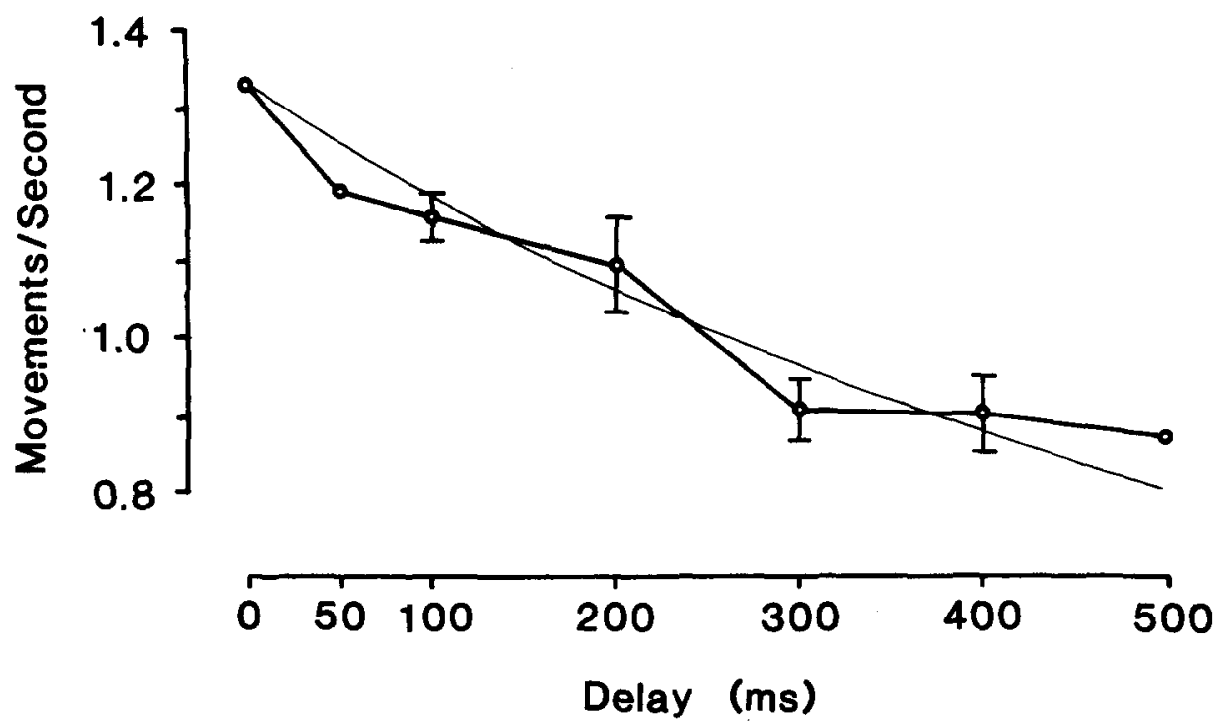

Fig. 4. Frequency of monkey's corrective movements (mean $\pm 1 \mathrm{SE}$ ) tracking sinusoids at six frequencies, normalised to the average base rate with immediate visual feedback of $1.33 \mathrm{~m} . \mathrm{p} . \mathrm{s}$. Delays in visual feedback reduced the average m.p.s. in close agreement with predicted values (light curve). 


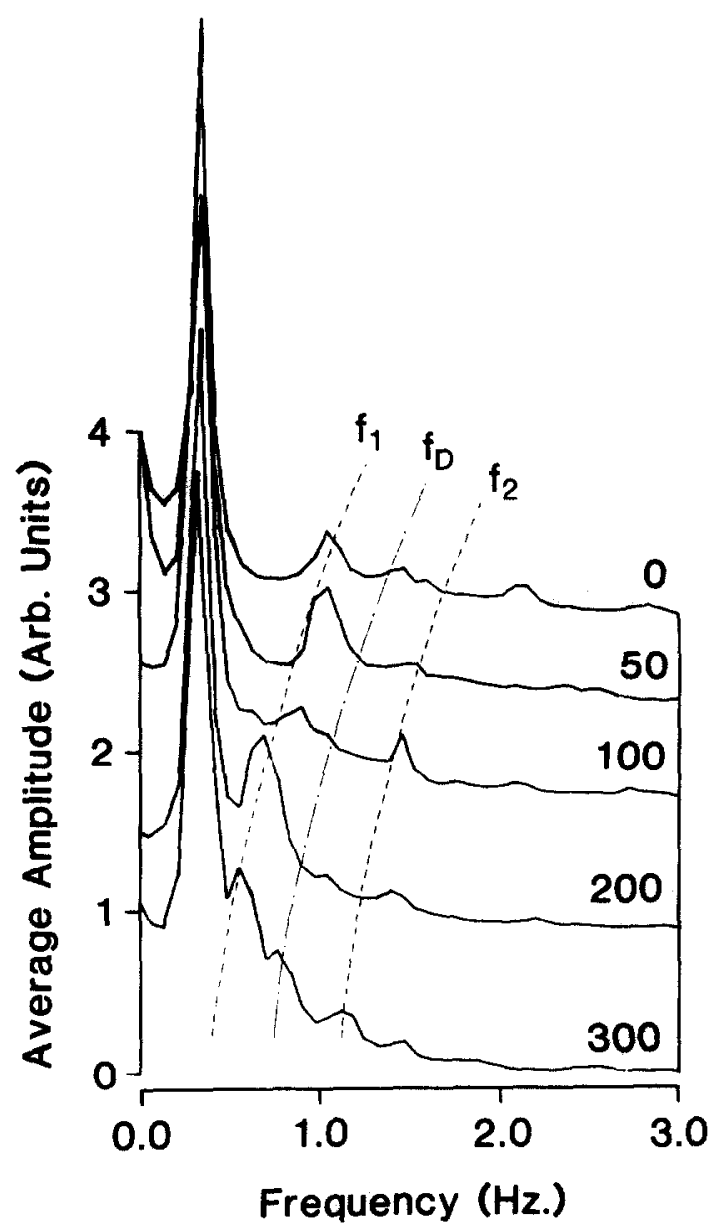

Fig. 5. A raster diagram of 5 averaged auto-regressive frequency spectra of the monkey's tracking responses to a target sinusoid of $0.35 \mathrm{~Hz}$. Each line is the average of $20-30$ individual spectra. The introduced delay in visual feedback is indicated to the right of each spectrum (milli-seconds). Predicted locations of components due to the corrective movements of the monkey are indicated by dotted lines (equation 3, Expcrimental Procedures).

programming a corrective movement and executing it all add to this minimum time (reviews see Refs 6, 11), until the average period between movements reached 750 and $\mathbf{4 4 2} \mathrm{ms}$ for monkey and human, respectively (i.e. 1.33 and 2.26 m.p.s.).

The monkey's rate of corrections was reduced dramatically by the introduction of delays in visual feedback of position. The fall in movement rate was approximately hyperbolic, and was closely fitted by equation 1 (Experimental Procedures). In the average frequency spectra, the step-and-hold form of the monkey's responses gave rise to a complex of peaks, representing the average frequency of corrective steps and two side bands. These components also showed the predicted shift towards lower frequencies as visual feedback was delayed.

Vicario and $\mathrm{Ghez}^{12}$ have reported a related experiment, in which cats were trained to use isometric force to return a displaced feeding tube to a central position. When the effects of their corrections were delayed by $60-180 \mathrm{~ms}$, the cats made secondary corrections one reaction time after first detecting the failure of the original response. This implies that the cats did not wait to assess the final outcome of their responses, but reacted as soon as the response error was apparent. However, their experimental design required a compensatory response to single step movements, whereas ours is a continuous tracking task. Thus, in our experiments the subjects may need to assess their current position before a correction is made. Both experiments do indicate, however, that the second response was triggered by visual feedback. These findings support our hypothesis that the frequency of positional corrections is not controlled by an asychronous sampling device, but is a consequence of the inevitable delays in the visuomotor feedback control loop.

Our proposed model of visuomotor feedback control ${ }^{7}$ requires that the flow of information around the loop is interrupted in some way. We suggested that the interruption was not due to the operation of an asynchronous sampling switch as has been previously suggested, ${ }^{3,9}, 13$ rather, we proposed that it results from subjects not bothering to correct for very small errors but waiting until they exceeded some critical threshold size (error dead-zone). The switching frequency of a sampling mechanism should be independent of visuomotor feedback loop time whereas that of a dead-zone type of non-linearity would be highly dependent on it. The fall in movement rate seen here under delayed feedback conditions therefore indicates that some form of dead-zone may be functional.

Craik ${ }^{3}$ argued against the idea that a dead-zone imposes intermittent operation of the visual feedback loop. He thought that this would mean that the amplitude of target movement would always govern the number of positional corrections, which is manifestly untrue. However, he neglected the role of loop

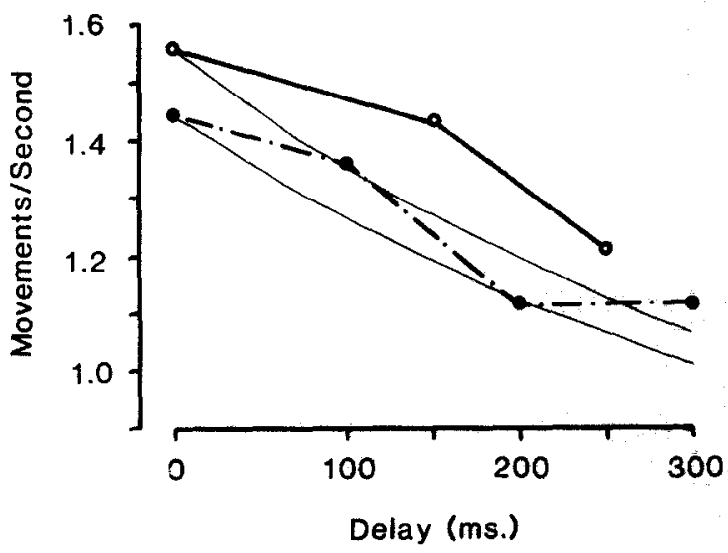

Fig. 6. Examples of fall in monkey's average rate of positional corrections when tracking a pseudorandom waveform with visual feedbelc delays (dark Whes), predicted rates of movements are given by the light lines. 


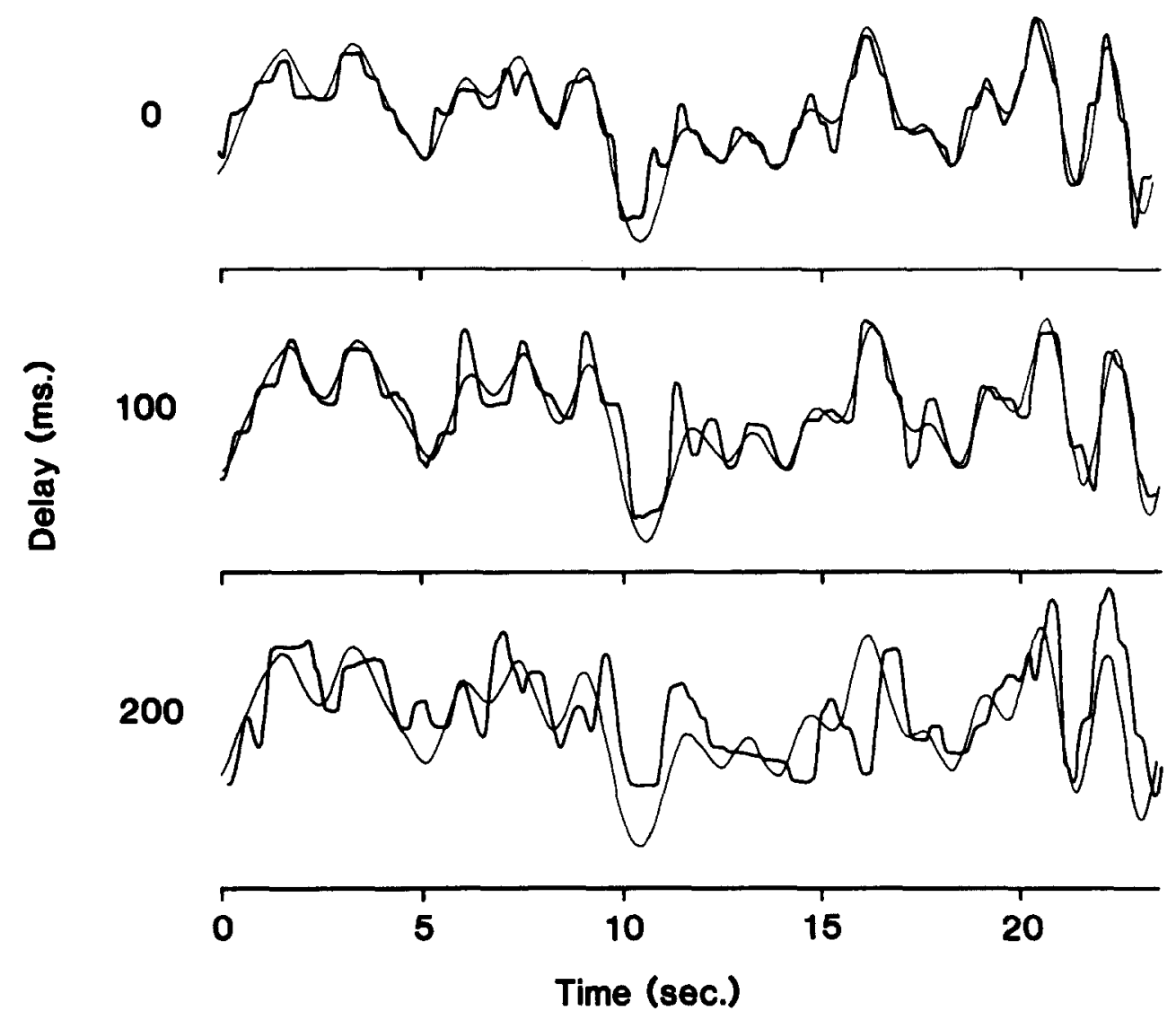

Fig. 7. Typical human responses (heavy lines) tracking a pseudo-random waveform (light lines). The normal frequency of step-and-hold movements (upper trace) was reduced by introducing 100 and $200 \mathrm{~ms}$ delays in visual feedback (middle and lower traces). The average amplitude of corrective movements also increased. 


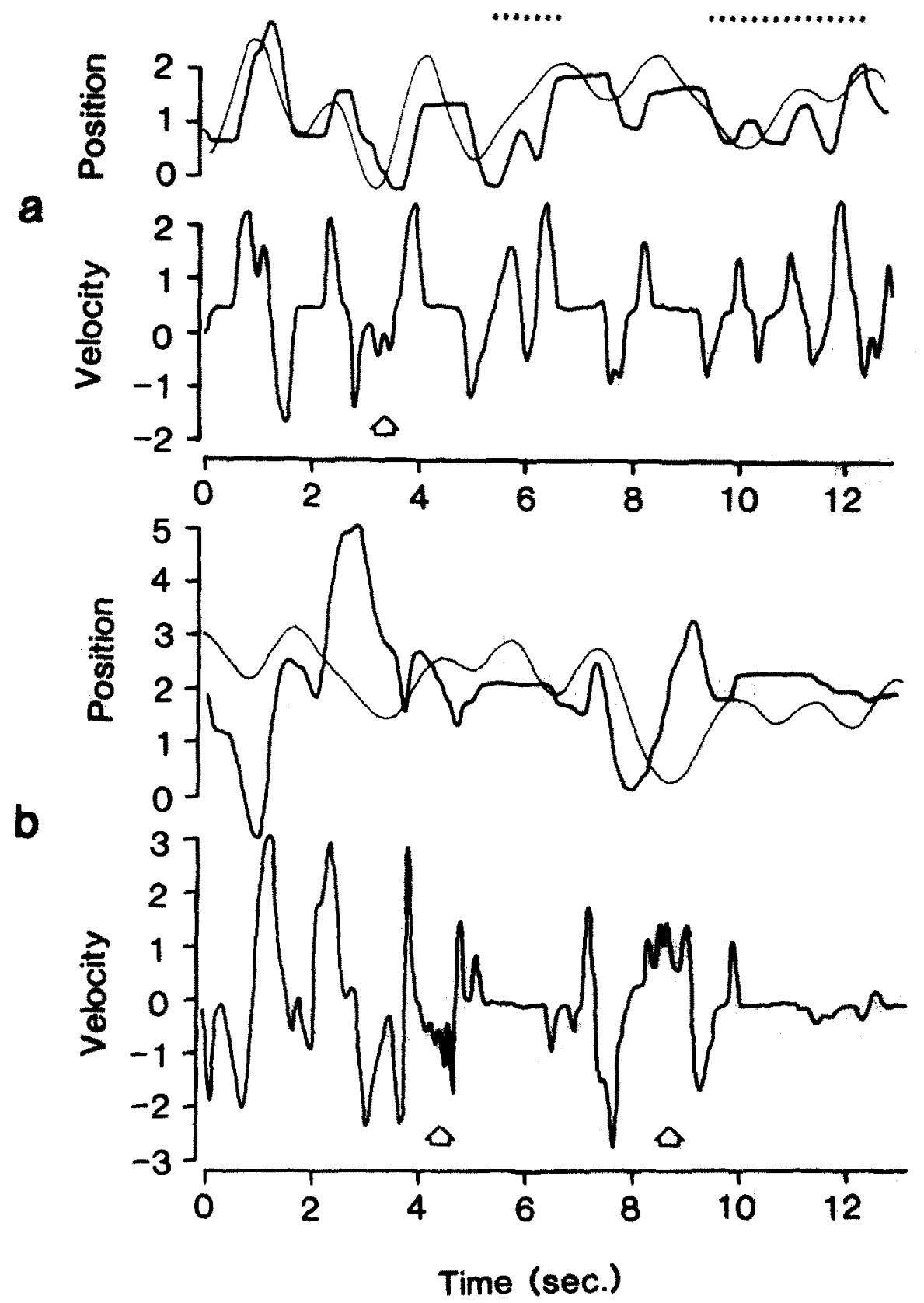

Fig. 8. Human responses (heavy lines) when tracking with loag visual feedback delays. a: Brief "hunting" (dotted periods) about the target waveform (light line); delay 200 ms. b: Btief petiods of hiph frepuency corrections (arrows); delay $300 \mathrm{~ms}$. 


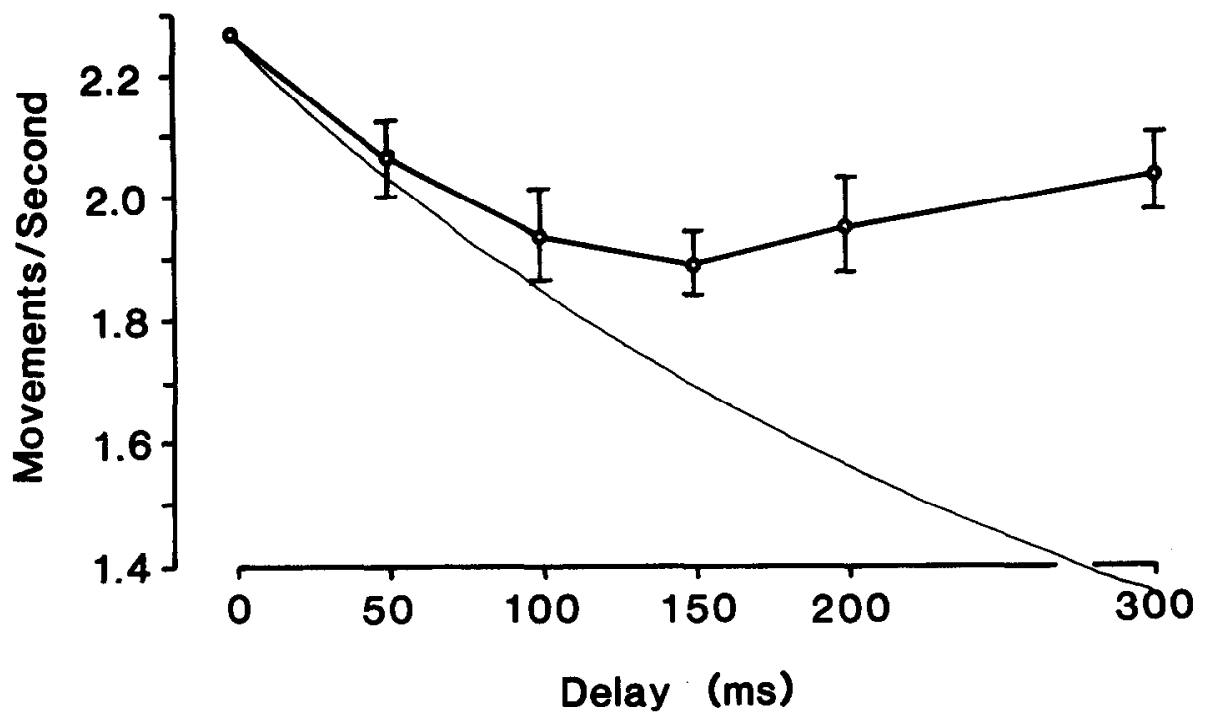

Fig. 9. Average frequency ( $\pm 1 \mathrm{SE}$ ) of corrective movements made by 5 human subjects tracking a peeudo-random waveform. The data have been normalised to the average base rate, as in Fig. 3. The average m.p.s. initially followed the predicted curve (light line), but increased with delays of over $150 \mathrm{~ms}$.

time. For very slow movements, the amplitude of target excursion may indeed determine the number of corrections because there is plenty of time for each one to be programmed and executed; hence, at low frequencies the number of corrections may be deadzone limited. ${ }^{8}$ As the frequency of target movement increases, however, so the visuomotor loop time becomes critical and at higher frequencies the rate of positional corrections becomes determined by loop time, as demonstrated by these results.

A major difference between the tracking responses of the monkey and of the human subjects was the humans' recovery of high correction rates when the feedback delay exceeded $150 \mathrm{~ms}$ (Fig. 9). The frequency of their movements varied considerably from moment-to-moment, however. The increase in average m.p.s. with longer delays was mainly a result of brief periods of very fast corrections of position (Fig. $8 \mathrm{~b})$. We believe that these periods indicate that humans adopt a new tracking strategy, in an attempt to overcome the problems of feedback control with long delays. When questioned about their tracking, most subjects felt that they improved their long-delay performance by attempting to mimic the movements of the target, rather than by attempting to correct the current positional error. It may be that humans tend to alternate between a feedback control strategy and one of open-loop feedforward control, where the rate of movement is no longer limited by feedback loop time. The monkey did not appear to adopt this strategy, however, and average m.p.s. was well predicted by the total feedback loop delay. We cannot say from the present data whether the feedforward pathway also displays intermittency, or whether it is based on, for example, feedforward of target position or velocity.

Acknowledgements-We are very grateful to Dr Kitney for his help, and for use of the facilities of the Engineering in Medicine Laboratory, and to P. Riddell for her assistance in these experiments. This work was supported by a grant from the Wellcome Trust; D.J.W. is supported by an M.R.C. studentship.

\section{REFERENCES}

1. Agarwal G. C. and Gottlieb G. L. (1971) Sampling in the human motor control system. IEEE Trans. Automat. Control AC16, 180-183.

2. Bekey G. A. (1962) The human operator as a sampled-data system. IEEE Trans. Human Factors Electron. HFE3, 43-51.

3. Craik K. J. W. (1947) Theory of the human operator in control systems. I-The operator as an engineering system. Br. J. Psychol. 38, 56-61.

4. Georgopoulos A. P., Kalaska J. F. and Massey J. T. (1981) Spatial trajectories and reaction times of aimed movements: effects of practice, uncertainty and change in target location. $J$. Neurophysiol. 46, 725-743.

5. Javid M. and Brenner E. (1963) Analysis, Transmission and Filtering of Signals. McGraw-Hill, London.

6. Keele S. W. (1981) Behavioural analysis of movement. In Handbook of Physiology: The Nervous System Vol. 2, pp. 1391-1414.

7. Kitney R. I., Miall R. C., Riddell P. M. and Stein J. F. (1984) Time series analysis of neuronal signals recorded in the cerebellum of trained monkeys. J. theor. Biol. 107, 367-385. 
8. Miall R. C., Weir D. J. and Stein J. F. (1984) Analysis of manual tracking movements in trained monkeys. Submitted for publication.

9. Navas F. and Stark L. (1968) Sampling or intermittency in hand control system dynamics. Biophys. J. 8, 252-302.

10. Pew P. W., Duffendack J. C. and Fensch L. K. (1967) Sine wave tracking revisited. IEEE Trans. Human Factors Electron. HFE8, 130-134.

11. Poulton E. C. (1981) Human Manual Control. In Handbook of Physiology: The Nervous System Vol. 2, pp. 1337-1389.

12. Vicario D. S. and Ghez C. (1984) The control of rapid limb movements in the cat. IV-Updating of ongoing isometric responses. Expl Brain Res. 55, 134-144.

13. Young L. R. and Stark L. (1963) Variable feedback experiments testing a sampled data model for eye tracking movements. IEEE Trans. Prof. Tech. Group Human Factors Electron. HFE4, 38-51. 